\title{
Many Commercially Available Antibodies for Detection of CHOP Expression as a Marker of Endoplasmic Reticulum Stress Fail Specificity Evaluation
}

\author{
Leena Haataja $\cdot$ Tatyana Gurlo $\cdot$ Chang-jiang Huang • \\ Peter C. Butler
}

Published online: 24 July 2008

(c) Humana Press 2008

\begin{abstract}
Endoplasmic reticulum (ER) stress contributes to beta cell death in type 2 diabetes (T2DM). ER stress is characterized by increased level of ER stress markers such as $\mathrm{C} / \mathrm{EBP}$ homologous protein (CHOP). Activation of CHOP leads to its translocation into the nucleus, where it induces cell death. We previously reported nuclear CHOP in pancreatic sections from T2DM, but not T1DM, and in human islet amyloid polypeptide (IAPP) transgenic rodent pancreatic sections. These studies underscore the importance of studying nuclear CHOP. We have observed inconsistency in the detection of CHOP antibodies reported in the literature and also in our own experiments. To investigate the specificity of CHOP antibodies, we first induced ER stress by tunicamycin in rat insulinoma (INS) cells and prepared nuclear and cytoplasmic fractions. Then we examined CHOP expression by Western blotting and immunocytochemistry using seven commercially available CHOP antibodies in INS cells and human IAPP (h-IAPP) transgenic rodent pancreatic tissue. These studies show that three commercially available CHOP antibodies out of seven tested were non-specific. In conclusion, we give recommendations for CHOP antibody selection and methods to verify CHOP antibody specificity. Also, we propose that the authors report the catalog and lot numbers of the CHOP antibodies used.
\end{abstract}

L. Haataja · T. Gurlo · C. Huang · P. C. Butler $(\bowtie)$ Larry Hillblom Islet Research Center, UCLA David Geffen School of Medicine, 900 Weyburn Place \#A, Los Angeles, CA 90024-2852, USA

e-mail: pbutler@mednet.ucla.edu
Keywords Endoplasmic reticulum stress - CHOP . Diabetes · Islet amyloid polypeptide

\section{Introduction}

Endoplasmic reticulum (ER) stress is an important pathway of the increased apoptosis in beta cells in type 2 diabetes (T2DM) [1-4] and neurons in neurodegenerative diseases. As such, it is a fast moving field with more than 1,000 citations in the last 2 years. One of the most commonly used indicators of ER stress is the increased expression and nuclear translocation of the transcription factor C/EBP homologous protein (CHOP) [5].

T2DM and neurodegenerative diseases share the characteristic of pathological formation of toxic oligomers of locally expressed amyloidogenic proteins, Alzheimers' beta protein in Alzheimers disease, synuclein in Parkinson's disease, and islet amyloid polypeptide (IAPP) in T2DM. IAPP is a $27-\mathrm{kDa}$ protein that is co-expressed and co-secreted with insulin. Human IAPP (h-IAPP) but not rodent IAPP (r-IAPP) is amyloidogenic. High expression rates of h-IAPP but not r-IAPP induces ER stress and apoptosis in rat insulinoma (INS) cells due to ER stress. Likewise high transgenic expression of h-IAPP but not r-IAPP in rodent beta cells induces apoptosis and ER stress $[2,6]$.

In studies of h-IAPP-induced beta cell apoptosis, we noted that commercially available antibodies for detection of CHOP by Western blotting and immunohistochemistry include several that are completely non-specific and others that vary from specific to non-specific from lot-to-lot. Given the large number of high profile papers in the ER stress field at present, we document this technical issue here for the benefit of other investigators. 


\section{Materials and Methods}

Cell Culture, Viral Transduction, and Tunicamycin

Treatment

Rat insulinoma INS 832/13 were cultured as described previously [6]. INS cells were plated on a $60-\mathrm{mm}$ tissue culture dish at a density of $3.0 \times 10^{6}$ cells/dish and cultured overnight. Cells were transduced with adenoviruses expressing human or rat preproIAPP-EGFP (h-IAPP or r-IAPP, respectively) or green fluorescein protein (GFP) at multiplicity of infection $(\mathrm{MOI})=100$ [2]. Tunicamycin (Sigma) was added to cells at $0.5 \mu \mathrm{g} / \mathrm{ml} 24 \mathrm{~h}$ after transduction. Eighteen hours later, cells were washed with PBS and collected by scraping in PBS. For a positive control, cells were treated with 1 or $5 \mu \mathrm{g} / \mathrm{ml}$ tunicamycin or nothing for $6 \mathrm{~h}$. For immunocytochemistry, INS cells were grown in Permanox Lab-Tek 8-well chamber slides (Nunc, Rochester, NY) and transduced with h-IAPP for $48 \mathrm{~h}$.

\section{Western Blot Analysis}

Nuclear and cytoplasmic fractions were extracted using the kit from Pierce according to the manufacturer's instructions (Pierce, Rockford, IL). Protein concentrations were determined using the BCA protein assay (Bio-Rad, Hercules, CA). About 10 or $5 \mu \mathrm{g}$ of cytoplasmic or nuclear protein, respectively, was separated on $4-12 \%$ Bis-Tris NuPAGE gels and blotted onto a PVDF membrane (Pall, Ann Arbor, MI). Membranes were probed with rabbit polyclonal or mouse monoclonal antibodies against $\mathrm{CHOP}$ (ab11419, Abcam, Cambridge, MA; sc-7351, sc-575 and sc-793, Santa Cruz, CA; G6916, Sigma), GAPDH, or PARP (Cell Signaling, Beverly, MA) as primary antibodies. Horseradish peroxidase-conjugated secondary antibodies $(1: 3,000)$ were from Zymed. Proteins were visualized using enhanced chemiluminescence (ECL, Millipore). CHOP was detected first, and then membranes were reused after stripping the primary antibody using Pierce stripping buffer. In order to get a good CHOP signal in a Western blot from rodent islet total protein lysates, we have combined one rabbit polyclonal and one mouse monoclonal CHOP antibody and performed detection using a mixture of rabbit and mouse secondary antibodies.

Transgenic Model and Immunocytochemistry

Human IAPP transgenic rat and mice were maintained and housed in accordance with Institutional Animal Care and Use Committee guidelines at UCLA. Pancreatic sections were prepared from $4 \%$ paraformaldehyde (EMS, Hatfield, PA) fixed tissue and immunostained as previously described $[2,6]$. For CHOP detection in INS cells, h-IAPP expressing
INS cells were fixed with $4 \%$ paraformaldehyde for $20 \mathrm{~min}$ at room temperature and stained for $\mathrm{CHOP}$ antibodies (see above). Slides were viewed under the fluorescent microscope DM6000 (Leica, Germany) using 20× objective.

\section{Results}

Tunicamycin-Induced CHOP Nuclear Translocation in INS Cells was Detected by Four Out of Seven CHOP Antibodies Tested

In an attempt to identify specific CHOP antibodies for Western blotting, we used cell fractionation in tunicamycin-treated INS cells. As shown in Fig. 1a, CHOP was detected in the nuclear fraction with four out of seven CHOP antibodies tested. This experiment demonstrated that nuclear fractionation should be used to test the specificity of CHOP antibodies.

Nuclear CHOP Expression in Pancreatic Tissue was Detected by Three Out of Seven Antibodies Tested

We have previously shown nuclear CHOP in pancreatic sections of type 2 diabetic humans and h-IAPP transgenic rodents $[2,6]$. As shown in Fig. 1b, not all CHOP antibodies detected nuclear CHOP. About $8.5 \pm 1.9 \%$ h-IAPP expressing INS cells were nuclear CHOP-positive using mouse monoclonal or rabbit polyclonal antibody. In mouse tissue, nuclear CHOP was detected only using rabbit antibody and the proportion of nuclear CHOP in beta cells was $0.8 \pm 0.1 \%$. None of the antibodies tested detected CHOP when frozen tissue sections were used.

\section{Conclusions}

We report that three out of seven commercially available CHOP antibodies tested gave false results by Western blotting and immunocytochemistry for detection of CHOP. Investigators using CHOP protein expression to establish the presence or absence of ER stress are strongly advised to establish the specificity of antibodies used, especially since we even observed a lot-to-lot variance in specificity from the same commercial source.

We advise that positive control experiments with a known inducer of ER stress, such as tunicamycin, are undertaken to confirm detection of a protein of the correct size $(29 \mathrm{kDa})$ in the nuclear fraction. We then advise use of a positive control for immunocytochemistry with the antibody that is successful for Western blotting, ensuring that the mode of fixation mirrors that is to be used in the test tissue (frozen vs. paraffin embedded). Finally, a new lot of CHOP antibody 


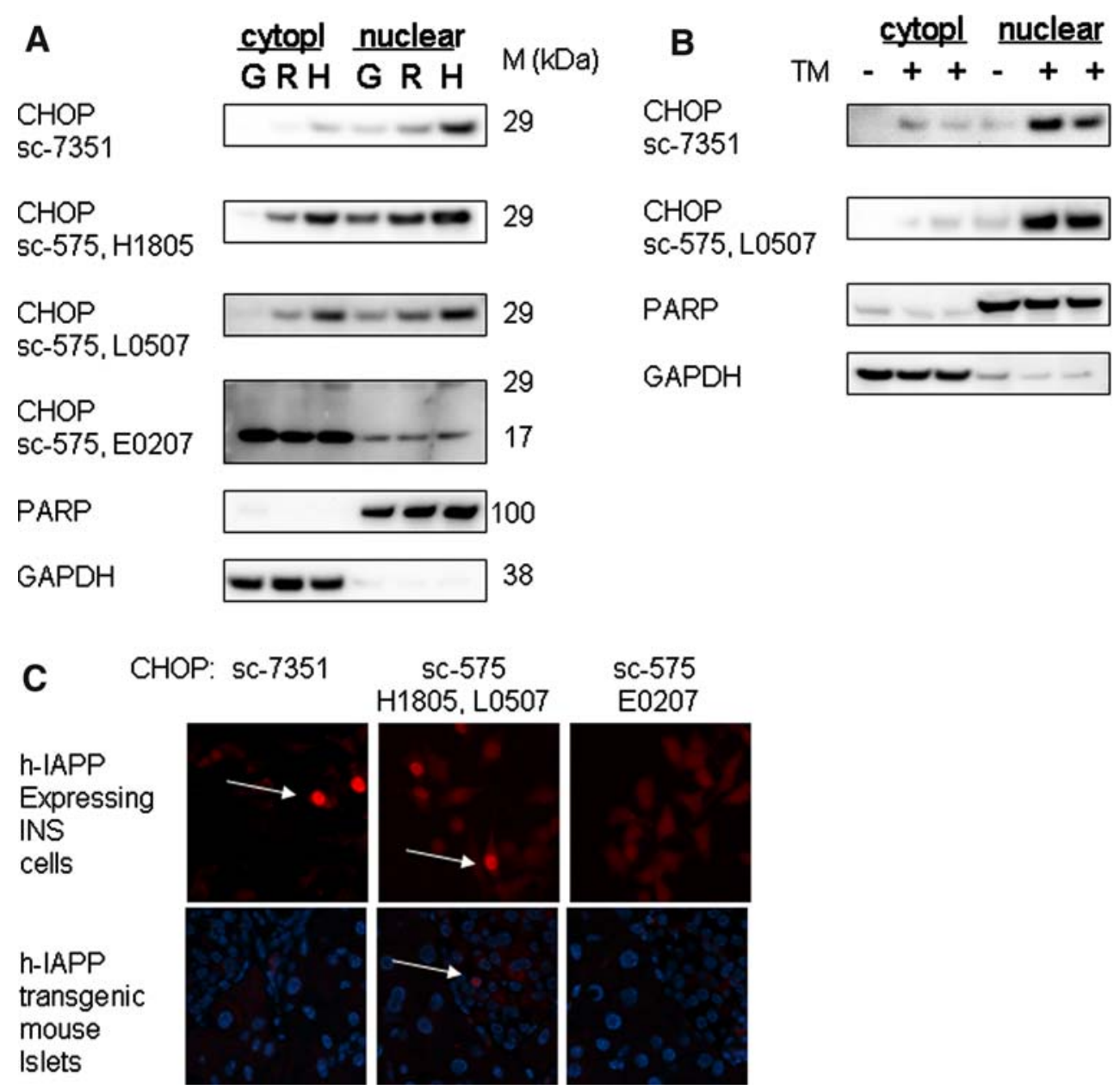

Fig. 1 The specificity of the CHOP antibodies. (a) INS 832/13 cells expressing GFP $(\mathrm{G})$, r-IAPP $(\mathrm{R})$, or h-IAPP $(\mathrm{H})$ were treated with $0.5 \mu \mathrm{g} / \mathrm{ml}$ tunicamycin and fractionated into cytoplasmic and nuclear fractions. (b) INS cells were cultured in the presence or absence of 1 or $5 \mu \mathrm{g} / \mathrm{ml}$ tunicamycin (TM) for $6 \mathrm{~h}$ and fractionated. Lysates were analyzed by immunoblotting using different CHOP antibodies. Positive staining was also obtained using $\mathrm{CHOP}$ antibody 9C8 (cat\#ab11419, Abcam). Non-specific signal in our testing conditions

even with the same catalog number that was previously used needs to be tested and compared with the results obtained with a previous antibody lot. Given these findings, it is also perhaps advisable for authors to affirm that these steps have been undertaken when reporting ER stress (or its absence) on the basis of nuclear CHOP detection.

Acknowledgments These studies were supported by the NIH (DK59579) and the Larry Hillblom Foundation. We thank Ryan Galasso for technical support.

\section{References}

1. Ozcan, U., Cao, Q., Yilmaz, E., Lee, A. H., Iwakoshi, N. N., Ozdelen, E., et al. (2004). Endoplasmic reticulum stress links obesity, insulin action, and type 2 diabetes. Science, 306(5695), $457-461$.

in INS cells was obtained using CHOP antibodies cat\#G6916 (lot\#57K4864, Sigma) and cat\#sc-793R-20 (lot\# F0105, Santa Cruz). The filters were also probed for PARP and GAPDH to show successful fractionation to nuclear and cytoplasmic fractions, respectively. (c) Human IAPP expressing INS cells (upper panel) and pancreatic sections of human IAPP transgenic mouse (lower panel) were stained with different CHOP antibodies. Positive staining was also obtained using CHOP antibody 9C8 (cat\#ab11419, Abcam)

2. Huang, C. J., Lin, C. Y., Haataja, L., Gurlo, T., Butler, A. E., Rizza, R. A., et al. (2007). High expression rates of human islet amyloid polypeptide induce endoplasmic reticulum stress mediated beta-cell apoptosis, a characteristic of humans with type 2 but not type 1 diabetes. Diabetes, 56(8), 2016-2027.

3. Laybutt, D. R., Preston, A. M., Akerfeldt, M. C., Kench, J. G., Busch, A. K., Biankin, A. V., et al. (2007). Endoplasmic reticulum stress contributes to beta cell apoptosis in type 2 diabetes. Diabetologia, 50(4), 752-763.

4. Marchetti, P., Bugliani, M., Lupi, R., Marselli, L., Masini, M., Boggi, U., et al. (2007). The endoplasmic reticulum in pancreatic beta cells of type 2 diabetes patients. Diabetologia, 50(12), 2486-2494.

5. Oyadomari, S., \& Mori, M. (2004). Roles of CHOP/GADD153 in endoplasmic reticulum stress. Cell Death and Differentiation, 11(4), 381-389.

6. Huang, C. J., Haataja, L., Gurlo, T., Butler, A. E., Wu, X., Soeller, W. C., et al. (2007). Induction of endoplasmic reticulum stressinduced-cell apoptosis and accumulation of polyubiquitinated proteins by human islet amyloid polypeptide. American Journal of Physiology, Endocrinology Metabalism, 293(6), E1656-E1662. 